\section{Selection of controls}

SIR: I read with interest the paper by Professor Morice (Cognitive inflexibility and pre-frontal dysfunction in schizophrenia and mania Journal, July $1990,157,50-54)$. To enable assessment of the generalisability of these results to other schizophrenic and manic groups, it would be of interest to know how the subjects in each group were chosen (e.g. randomly? every $n$th patient presenting? on some representational basis? etc.).

I commend the attempt to select a "control group with a mean IQ lower than that usually obtained for control groups in psychiatric research", although this endeavour was unsuccessful in this instance! This is a problem that recurs throughout the literature pertaining to the Wisconsin Card Sorting Test in my opinion. Although a significant correlation between WCST results and IQ or educational level has not been reported, the trend seems always to be in the expected direction. That is, the putatively deficient group (viz. focal frontal, schizophrenic, etc.), in almost every case has a lower mean IQ or fewer years of education than the control group to which it is compared. Thus, for example, Heaton (1981) provides the following data: focal frontal only - IQ 95.5, focal non-frontal only - IQ 101.5, and normals - IQ 114.0; Weinberger et al (1986): schizophrenics - 12.5 years of education; controls - 16.8 years, and more recently, Miller et al (Journal, January 1991, 158, 76-82) with late life psychosis (LLP) patients: LLP IQ 84.5, controls - 109.5.

On the basis of these results, let us assume for the purpose of argument that overall IQ does impinge on WCST performance. Then looking at the results presented by Professor Morice, we find that for the schizophrenic patients, mean number of categories completed (3.1) is 1.6 standard deviations (s.d.) below the mean for the controls (5.2), a reasonable result considering that their mean IQ (84.5) is 1.4 s.d. below the mean for the controls (103.7). However, manic patients, whose mean number of categories completed (3.3) is similarly low (1.5 s.d. below the control mean) do not show such a discrepancy in their IQ results (mean IQ $=97.3$, only 0.46 s.d. below the mean for the controls)! An interpretation of these results might be that, relative to their overall functioning level as measured by the full scale $I Q$, the manic patients show deficiencies on the WCST while the schizophrenic patients do not!

I would also make the observation that while $67 \%$ and $65 \%$ respectively of schizophrenic and manic patients showed these deficits, $33 \%$ and $35 \%$ respectively did not. It would be interesting to know the characteristics of these groups of patients (e.g. did the latter group of schizophrenics demonstrate increased incidence of auditory hallucinations, which might indicate a temporal rather than frontal focus etc.?).

\section{Morisset Hospital NSW, Australia}

IAN GALE

\section{References}

HeAton, R. K. (1981) A Manual for the Wisconsin Card Sorting Test. Odessa, Florida: Psychological Assessment Resources.

Weinberger, D. R., Berman, K. F. \& ZeC, R. F. (1986) Physiologic dysfunction of dorsolateral prefrontal cortex in schizophrenia. I. Regional cerebral blood flow evidence. Archives of General Psychiatry, 43, 114-124.

AUTHOR's REPLY: Subjects were selected consecutively from the subgroups as described in the original paper. Although $\mathrm{Mr}$ Gale asserts that the attempts to select a control group with a mean IQ lower than that usually obtained for control groups were unsuccessful, his own examples belie this assertion:

$\begin{array}{ccc}\text { Heaton } & \text { Miller et al } & \text { Morice } \\ (1981) & (1991) & (1990) \\ 114.0 & 109.5 & 103.7\end{array}$

$\mathrm{Mr} \mathrm{Gale}$ is right to be intrigued by the relationship between general intelligence and performance on the Wisconsin Card Sorting Test. A dissociation did appear between the schizophrenic and manic/control groups. As he points out, the general consensus to date has been that while WCST performance can be impaired in the presence of average or above-average IQ, IQ per se does not affect WCST performance to any marked degree. This seems to be supported by results for the manic and control groups.

That schizophrenics demonstrated both low mean IQ and mean impaired WCST performance does not, of course, mean that low IQ only causes poor WCST performance, which $\mathrm{Mr}$ Gale offers as his alternative explanation. While theoretically this could be so, I can find no evidence in the literature to support it.

Rather, and to me a more persuasive explanation, the schizophrenic 'process' (whatever that may be) appears to result in a reduction both of measured IQ and of WCST performance. The manic 'process' appears, relatively at least, to spare IQ. These differences could possibly result from laterality differences in pre-frontal functioning in the two disorders.

Also, they could result from group differences in pre-morbid IQ. Unfortunately, a measure of pre-morbid IQ, such as the New Adult Reading Test (NART), was not used in this study. 